\title{
Role of rehabilitation in a COVID-19 survivor with intensive care unit-acquired weakness: A case report
}

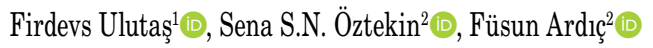 \\ ${ }^{1}$ Department of Internal Medicine, Division of Rheumatology, Pamukkale University Faculty of Medicine, Denizli, Turkey \\ ${ }^{2}$ Department of Physical Medicine and Rehabilitation, Pamukkale University Faculty of Medicine, Denizli, Turkey
}

Received: September 23, 2020 Accepted: February 04, 2021 Published online: March 04, 2021

\begin{abstract}
Intensive care unit-acquired weakness (ICU-AW) is a catastrophic and debilitating clinical condition that causes generalized weakness and predisposes to adverse short- and long-term outcomes. Novel coronavirus disease-2019 (COVID-19) has been a global pandemic since November 2019. Each additional ICU admission for COVID-19 stresses intensive care unit (ICU) and hospital capacity. Accurately designed, timely rehabilitation procedures may reduce the patient burden of ICUs and hospitals. In this article, we describe a COVID-19 survivor who developed acute respiratory distress syndrome (ARDS) and ICU-AW. Early pulmonary rehabilitation in the ICU and long-term rehabilitation maintenance after the ICU and hospital discharge resulted in a positive outcome.
\end{abstract}

Keywords: COVID-19, intensive care unit-acquired weakness, rehabilitation.

Severe acute respiratory syndrome-coronavirus-2 (SARS-CoV-2) has become a major public health issue since November 2019. The highly transmissible SARS-CoV-2 has brought a huge burden to health care systems. In a comprehensive systematic review, $20.3 \%$ of patients with novel coronavirus disease-2019 (COVID-19) require intensive care unit (ICU) admission and almost $75 \%$ of patients with COVID-19 admitted to the ICU need invasive mechanical ventilation (IMV). ${ }^{[1]}$ Bhatraju et al. ${ }^{[2]}$ identified 24 critically ill patients with COVID-19, and the minimum length of stay in the ICU was 14 days for each patient.

Intensive care unit-acquired weakness (ICU-AW) is a catastrophic and debilitating clinical condition that occurs via complex structural/functional changes within myofibers and neurons. The clinical presentation may be neuropathic, myopathic, or a combination of both. ${ }^{[3]}$ Typically, ICU-AW occurs as a consequence of critical illness and is primarily associated with loss of muscle protein. The main pathogenetic mechanisms associated with muscle protein catabolism have been described including an unsteady ubiquitin proteasome system and dysregulated autophagy. ${ }^{[4]}$ In addition, peripheral nervous system involvement after COVID-19 pneumonia has been explained by inflammatory and/or immune-mediated neuromuscular damage. ${ }^{[4]}$ Recently, a small-sized cohort including 14 patients with generalized muscle weakness after COVID-19 pneumonia was published and the vast majority of the patients were more likely to have axonal and asymmetric polyneuropathy. ${ }^{[5]}$ Current guidelines recommend principally evaluation of muscle strength for diagnosis. The diagnosis of ICU-AW includes the following criteria: (i) Medical Research Council (MRC) sum score of $<48$ for muscle strength evaluation; (ii) disturbed neuromuscular function characterized by a hand-held dynamometer and hand grip strength; and (iii) decreased respiratory muscle strength. ${ }^{[6]}$ Intensive

Corresponding author: Füsun Ardıç, MD. Pamukkale Üniversitesi Tıp Fakültesi Fiziksel Tıp ve Rehabilitasyon Anabilim Dalı, 20070 Kınıklı, Denizli, Türkiye.

e-mail: ardicf@gmail.com

Ulutaş F, Öztekin SSN, Ardı̣ F. Role of rehabilitation in a COVID-19 survivor with intensive care unit-acquired weakness: A case report. Turk J Phys Med Rehab 2021;67(1):115-119. 
care unit-acquired weakness leads to a delay in weaning and hospital discharge. It is also associated with higher in-ICU, in-hospital and post-ICU mortality and low physical function at six months after ICU discharge. ${ }^{[7]}$

Although ICU-AW is an increasingly recognized problem, there is no research or available data related to the incidence and prevalence of ICU-AW post-COVID-19. It is also not clear whether these patients require more specific rehabilitation programs in the ICU setting. The long-term quality of life among COVID-19 survivors is also unknown. In this article, we emphasize the important role of rehabilitation in describing a critically ill COVID-19 patient who developed acute respiratory distress syndrome (ARDS) and ICU-AW. We believe that this paper is quite valuable due to being the first published case related to rehabilitation in patients with ICU-AW from Turkey.

\section{CASE REPORT}

A 48-year-old man who was previously obese and had non-alcoholic steatohepatitis, was diagnosed with SARS-CoV-2 infection at the beginning of March 2020 based on the presence of both a ground-glass pattern on thoracic computed tomography (CT) and positive nasopharyngeal swab test for viral ribonucleic acid. He was immediately isolated in the Department of Infectious Diseases, and oseltamivir, hydroxychloroquine and broad-spectrum antibiotics were started. On the third day of treatment, he underwent endotracheal intubation and IMV in the ICU due to respiratory failure, and favipiravir was added to the treatment. A rehabilitation program could not be implemented due to ARDS, following the recommendations of the Turkish Society of Physical Medicine and Rehabilitation for COVID-19 patients in Turkey ${ }^{[8]}$ As soon as cardiopulmonary stability was provided and SARS-CoV-2 negativity was confirmed, the patient underwent the following rehabilitation interventions: (i) in-bed positioning every $2 \mathrm{~h}$ to avoid decubitus ulcers; (ii) passive range of motion (ROM) to prevent contractures due to unconsciousness and staying on mechanical ventilation; (iii) using prone position 12 to $16 \mathrm{~h}$ per day that supports more oxygenation. During follow-up, the active rehabilitation program was interrupted for more than two weeks as a consequence of cytokine storm syndrome and candidemia. Different treatment modalities consisting of intravenous tocilizumab, immune plasma, intravenous high-dose glucocorticoids, and antifungal therapy were administered for this severe clinical condition. After clinical stability and weaning were provided, controlled breathing techniques (pursed-lips breathing, diaphragmatic, slow and deep breathing) together with bronchial hygiene-airway clearance techniques (controlled cough maneuver, huffing, incentive spirometry and postural drainage) were added to the former rehabilitation program in the ICU. A home-based respiratory muscle exercise program with a threshold inspiratory muscle trainer (IMT) device was planned for the patient.

The patient was admitted to the inpatient clinic of the Department of Physical Medicine and Rehabilitation (PMR) after a total of 71 days in the ICU. He had weakness predominantly involving distal muscles of the upper and lower extremities including right wrist and bilateral foot drop in addition to symmetrical mild distal muscle atrophy on physical examination. Contractures of the left hip and left knee and a sacral decubitus ulcer with a size of $5 \times 5 \mathrm{~cm}$ were also detected. Electroneuromyography showed sensorimotor axonal peripheral neuropathy. Cardiopulmonary functions could not be assessed by exercise tests and no respiratory function tests were able to be performed due to the possible spread risk of SARS-CoV-2 and the physical impairment of the patient. Thoracic CT and echocardiography did not reveal any new findings including consolidation, ground-glass infiltration, heart failure, or heart valve pathologies. His biochemical analysis revealed normal ranges of acute phase reactants, serum electrolytes, liver and kidney function tests, except for mild hypoalbuminemia and isolated low creatinine levels that may be attributed to catabolic processes and muscle atrophy. After secondary causes were ruled out, a diagnosis of ICU-AW originating from critical illness polyneuropathy (CIP) was confirmed based on all of the clinical and neurophysiological findings.

At the beginning of the planned rehabilitation program, the Mini-Mental State Examination (MMSE) score was 25 and his General Health Perception score was 5. The Mental Health Perception and Physical Function scores were 0 as assessed by the Short Form-36 (SF-36). His functional ambulation category (FAC) level was calculated as zero (FAC level: 0), while his muscle strength score was 30 according to MRC. Due to postural hypotensive attacks, he was gradually trained on the tilt table for one week. In addition, active-assisted and active ROM (providing full ROM in all possible directions for cervical, shoulder, elbow, hip, knee and ankle), isometric (starting from $5 \mathrm{sec}$ with increasing times depending on the fatigue of the patient) and isotonic strength exercises (three sets gradually with Theraband and different weights, particularly 


\begin{tabular}{|c|c|c|c|c|}
\hline MRC & d CMT res & $\begin{array}{l}\text { CABLE } 1 \\
\text { he first and e }\end{array}$ & eks of PMI & \\
\hline \multirow{3}{*}{ Week in PMR service } & \multicolumn{2}{|c|}{ MRC (score/5) } & \multicolumn{2}{|c|}{ CMT (Newton) } \\
\hline & First week & Eighth week & First week & Eighth week \\
\hline & Right/left & Right/left & Right/left & Right/left \\
\hline Shoulder abduction & $3 / 3$ & $4 / 4$ & $49.8 / 58.6$ & $83.6 / 106.6$ \\
\hline Elbow flexion & $3 / 3$ & $5 / 5$ & $71.1 / 117$ & $157.6 / 231.3$ \\
\hline Wrist extension & $1 / 3$ & $3 / 5$ & WD/71.8 & $8.8 / 117$ \\
\hline Hip flexion & $3 / 3$ & $5 / 5$ & $114 / 132.3$ & $142.3 / 154.3$ \\
\hline Knee extension & $3 / 3$ & $5 / 5$ & $82.1 / 78.46$ & $170.3 / 160.6$ \\
\hline Ankle dorsiflexion & $1 / 1$ & $2 / 1$ & FD & FD \\
\hline
\end{tabular}

for muscles of the deltoideus, triceps, biceps, hip flexors, quadriceps and hamstrings, gastrocnemius) and efforts for mobilization were applied to the patient. He received a physical rehabilitation program $2 \mathrm{~h}$ daily for five days a week accompanied by a physiotherapist for a total of 60 days. Due to his widespread muscle weakness and immobilization, methenolone enanthate (Primobolan Depot) with doses of $400 \mathrm{mg} /$ week was also administered five times at varying frequencies, to take advantage of its anabolic effects. ${ }^{[9]}$ The patient did not experience any cardiopulmonary complications or exercise-induced desaturation during these physical rehabilitation procedures. Neuromuscular electrical stimulation (frequency: $100 \mathrm{pps}$, stimulus cycle: 10/50, $20 \mathrm{~min}$, two times, five days a week, with the Chattanooga Intelect $^{\star}$ Mobile Stim 2777, Chattanooga Medical Supply, TN, USA) was performed for muscle groups that had a MRC score of $<3$. Collagen-containing dressing was used for the sacral decubitus ulcer in addition to regular, appropriate in-bed positioning. The patient was able to walk with a high-density ankle-foot orthosis for the bilateral drop foot and walker (FAC level: 2). A significant improvement was observed in the cognitive scores according to the MMSE and the quality of life and life activity scales according to SF-36 at his discharge (MMSE: 29 points, General Health Perception: 30 points, Mental Health Perception: 48 points, and Physical Function parameters: 0 point).

Measurements of the muscle strength and evaluation of fine motor grip skills including hand grip and pinch strength tests at the first and eighth week of PMR admission are shown in Tables 1 and 2. Hand grip and pinch strength measurements were obtained by using a hydraulic hand dynamometer $(0-90 \mathrm{~kg}$, North
Coast Medical, CA, USA) and a hydraulic pinch gauge (0-42.5 kg, BASELINE Corp., NY, USA), respectively. The hand grip and pinch positions were also applied as described by Mathiowetz et al. ${ }^{[10]}$

The manual muscle testing using the MRC scale was roughly used as a suggested standard tool for diagnosis of ICU-AW in our patient. However, hand-held dynamometry was preferred to provide reliable, measurable global strength and to assess and monitor strength recovery in the longitudinal followup time. We reported the MMT measurements which were higher than 3, but not fulfilling 4 as 3 on the MRC scale. We also measured minimal detectable resistance by hand grip dynamometry to obtain more objective data. The test positions for hand-held dynamometer were reported by Bohannon ${ }^{[1]}$ in 1997. Recently, the use of hand-held dynamometry was reported as a reliable tool in critically ill patients who had powerful enough strength to overcome gravity (MRC $\geq 3$ ) in lower and upper muscles. ${ }^{[12]}$ However, in that study, the knee extension muscle strength position was modified in these patients. In our case, we used these modified

\section{TABLE 2}

Hand grip strength and pinch strength measurements $(\mathrm{kg})$ for fine motor grip skills at the first and eighth weeks of PMR service

\begin{tabular}{|lccc|}
\hline Week in PMR service & First week & & Eighth week \\
\cline { 2 - 2 } Localization & Right/left & & Right/left \\
\hline Grip strength & $3 / 23$ & & $10 / 29$ \\
Lateral pinch & $3 / 6$ & & $6 / 8.5$ \\
Tip pinch & $0 / 4$ & & $2.5 / 4.5$ \\
Palmar pinch & $0 / 6$ & $6 / 7.5$ \\
\hline PMR: Physical medicine and rehabilitation. & & \\
\hline
\end{tabular}


test positions accordingly. A written informed consent was obtained from the patient.

\section{DISCUSSION}

In the present case, we emphasize a favorable prognosis for a COVID-19 survivor with ICU-AW originating from CIP. Early and appropriate pulmonary and physical rehabilitation interventions improved the quality of our patient's life and functional capacity. We also show that these rehabilitation procedures could be implemented safely without the increased mortality for COVID-19 patients with acute illness or long-term follow-up periods.

Recently, the increased length of ICU stay, prolonged IMV, use of neuromuscular blockers and glucocorticoids, prolonged immobility, sepsis and comorbidities such as diabetes mellitus and renal failure have been described as the risk factors for ICU-AW. ${ }^{[13]}$ Besides all these proven risk factors, Montalvan et al. ${ }^{[14]}$ emphasized that SARS-CoV-2 was a new neuropathogen with its receptor being widely expressed. It may have harmful immunemediated effects on myofibrils. Nevertheless, neurological complications of COVID-19 commonly occur through concurrent critical illness and how COVID-19 makes patients susceptible to neuromuscular damage remains unclear. Insulin resistance, IMV, using high-dose glucocorticoids, prolonged ICU stay, and ARDS were all culprit factors in our patient.

The favorable prognosis of our patient can be attributed to merits of early rehabilitation and multidisciplinary team work under the supervision of a PMR physician. Rehabilitation should be planned in a patient-specific manner, based on current functional and cognitive status and the patient's comorbidities. ${ }^{[15]}$ Rehabilitation procedures should be progressed gradually with both in-bed and out-of-bed exercises. Simple and reproducible rehabilitation interventions including passive, active-assisted, active or resisted joint ROM, cycle ergometry, therapeutic exercises, functional mobility and, finally, occupational activities are critical. Passive ROM, passive cycling, and electrical muscle stimulation are important parts of early rehabilitation in the admission period. Strengthening, endurance, and balance should be aimed in the follow-up programs. ${ }^{[16]}$ All interventions, with support from previous literature and guidelines, were successfully carried out in our patient without any special approach or any complication. Another important point is related to anabolic steroids. Their additive role in improving muscle mass and strength has been reported and should be kept in mind. ${ }^{[17]}$ Methenolone enanthate has been safely used for a short time as a pharmacological agent in our patient.

Unfortunately, there is still no validated preventive interventions for developing ICU-AW. Avoiding hyperglycemia and minimizing sedation and neuromuscular electrical stimulation are known to having remarkable benefits. ${ }^{[18]}$ Non-invasive methods rather than IMV should be chosen in these patients due to barotrauma, longer ICU stay, and even death. ${ }^{[19]}$ Early pulmonary rehabilitation including controlled respiration and airway clearance techniques, and strengthening of inspiratory muscles are of utmost importance to maintain and improve lung capacity. ${ }^{[20]}$ In addition, appropriate positioning in bed prevents long-term contractures and pressure injuries.

Prior to hospital discharge, tele-rehabilitation services or home-based exercise programs should be explained in detail to each patient in practice. ${ }^{[21]}$ Another important intervention at hospital discharge is comprehensive education of the patient on proper nutrition for muscle remodeling. In addition, appropriate diets may prevent subsequent development of obesity which is a well-known risk factor for impaired lung function. ${ }^{[22]}$

In conclusion, available data on long-term results and extent of damage are still insufficient in COVID-19 patients. Early physical and pulmonary rehabilitation programs may shorten hospital and ICU stay and improve long-term complications. Critically ill COVID-19 survivors may experience long-term poor outcomes including physiological, cognitive, and mental impairment. The PMR physicians and timely rehabilitation play a key role in improving functional capacity and limiting disability in this pandemic, fighting against COVID-19 in all aspects of supportive care, including nutrition, posture, airway, oxygen supplementation, airway clearance techniques, pulmonary exercises, strengthening and physical activity.

\section{Declaration of conflicting interests}

The authors declared no conflicts of interest with respect to the authorship and/or publication of this article.

Funding

The authors received no financial support for the research and/or authorship of this article. 


\section{REFERENCES}

1. Rodriguez-Morales AJ, Cardona-Ospina JA, GutiérrezOcampo E, Villamizar-Peña R, Holguin-Rivera Y, EscaleraAntezana JP, et al. Clinical, laboratory and imaging features of COVID-19: A systematic review and meta-analysis. Travel Med Infect Dis 2020;34:101623.

2. Bhatraju PK, Ghassemieh BJ, Nichols M, Kim R, Jerome KR, Nalla AK, et al. Covid-19 in Critically Ill Patients in the Seattle Region - Case Series. N Engl J Med 2020;382:201222.

3. Vanhorebeek I, Latronico N, Van den Berghe G. ICUacquired weakness. Intensive Care Med 2020;46:637-53.

4. Lad H, Saumur TM, Herridge MS, Dos Santos CC, Mathur S, Batt J, et al. Intensive care unit-acquired weakness: Not just another muscle atrophying condition. Int J Mol Sci 2020;21:7840.

5. Di Pietro DA, Luisa A, Vitacca M. Patients recovering from COVID-19 pneumonia at a sub-acute admissions unit exhibit profound muscular weakness: a case series. Eur J Phys Rehabil Med 2020. [Online ahead of print]

6. Hermans G, Van den Berghe G. Clinical review: intensive care unit acquired weakness. Crit Care 2015;19:274.

7. Wieske L, Dettling-Ihnenfeldt DS, Verhamme C, Nollet F, van Schaik IN, Schultz MJ, et al. Impact of ICU-acquired weakness on post-ICU physical functioning: a follow-up study. Crit Care 2015;19:196.

8. Kurtaiş Aytür Y, Köseoğlu BF, Özyemişçi Taşkıran Ö, Ordu-Gökkaya NK, Ünsal Delialioğlu S, Sonel Tur B, et al. Pulmonary rehabilitation principles in SARSCOV-2 infection (COVID-19): A guideline for the acute and subacute rehabilitation. Turk J Phys Med Rehabil 2020;66:104-20.

9. Woerdeman J, de Ronde W. Therapeutic effects of anabolic androgenic steroids on chronic diseases associated with muscle wasting. Expert Opin Investig Drugs 2011;20:87-97.

10. Mathiowetz V, Weber K, Volland G, Kashman N. Reliability and validity of grip and pinch strength evaluations. J Hand Surg Am 1984;9:222-6.
11. Bohannon RW. Reference values for extremity muscle strength obtained by hand-held dynamometry from adults aged 20 to 79 years. Arch Phys Med Rehabil 1997;78:26-32.

12. Vanpee G, Segers J, Van Mechelen H, Wouters P, Van den Berghe G, Hermans G, et al. The interobserver agreement of handheld dynamometry for muscle strength assessment in critically ill patients. Crit Care Med 2011;39:1929-34.

13. Appleton R, Kinsella J. Intensive care unit-acquired weakness. Continuing Education in Anaesthesia Critical Care \& Pain 2012;12:62-6.

14. Montalvan V, Lee J, Bueso T, De Toledo J, Rivas K. Neurological manifestations of COVID-19 and other coronavirus infections: A systematic review. Clin Neurol Neurosurg 2020;194:105921.

15. Anekwe DE, Biswas S, Bussières A, Spahija J. Early rehabilitation reduces the likelihood of developing intensive care unit-acquired weakness: a systematic review and metaanalysis. Physiotherapy 2020;107:1-10.

16. McNeary L, Maltser S, Verduzco-Gutierrez M. Navigating coronavirus disease 2019 (COVID-19) in Physiatry: A CAN report for inpatient rehabilitation facilities. PM R 2020;12:512-5.

17. Kuhn CM. Anabolic steroids. Recent Prog Horm Res 2002;57:411-34.

18. Zorowitz RD. ICU-acquired weakness: A rehabilitation perspective of diagnosis, treatment, and functional management. Chest 2016;150:966-71.

19. McGuinness G, Zhan C, Rosenberg N, Azour L, Wickstrom $\mathrm{M}$, Mason DM, et al. Increased incidence of barotrauma in patients with COVID-19 on invasive mechanical ventilation. Radiology 2020;297:E252-E262.

20. Hashem MD, Nelliot A, Needham DM. Early mobilization and rehabilitation in the ICU: moving back to the future. Respir Care 2016;61:971-9.

21. Hosey MM, Needham DM. Survivorship after COVID-19 ICU stay. Nat Rev Dis Primers 2020;6:60.

22. Finer N, Garnett SP, Bruun JM. COVID-19 and obesity. Clin Obes 2020;10:e12365. 